P125 presented in PS04: Poster teaser: Gait, technical and upper-limb

\title{
Effect of taping on 3-dimensional scapular kinematics and trapezius activity
}

\author{
Ana Clemente ${ }^{1, *}$, Marco Jardim ${ }^{1}$, Filomena Carnide ${ }^{2}$, Ricardo Matias ${ }^{1,2}$ \\ ${ }^{1}$ Escola Superior de Saúde, Instituto Politécnico de Setúbal, Setúbal, Portugal \\ ${ }^{2}$ Universidade de Lisboa, Faculdade de Motricidade Humana, Neuromechanics Research Group - CIPER, Lisboa, Portugal
}

Introduction: Patients with shoulder pain frequently present scapula dyskinesis that is believed to compromise shoulder stability and performance especially during dynamic activities. The current best available evidence points for restoring the imbalanced created by the lower trapezius (LT) and serratus anterior weakness, and upper trapezius (UT) hyperactivity for optimal scapular position and motion [1]. It is theorised in the literature that scapular taping (ST) contribute to an inhibition of the UT and a facilitation of the LT, and consequently, to an optimal scapular kinematics [2]. To the authors' best knowledge there are no studies describing ST effects on simultaneously measured trapezius activity and scapulothoracic 3D kinematics.

Research question: Does ST contributes to an inhibition of the UT and a facilitation of LT, and induces an optimal scapular kinematics in healthy subjects while executing two upper extremity functional tasks?

Methods: Twenty-two healthy individuals were examined with surface electromyography collected from the UT and LT fibres, and 3D kinematics of the thorax, scapula and humerus reconstructed with the use of an electromagnetic system, during two upper extremity tasks-slow full shoulder flexion and a fast ball throw. The order of the tasks' execution was randomly assigned, and both executed 3 times with and without ST. The taping was applied by the same researcher who is an experienced musculoskeletal physiotherapist. Muscle's onset and activation intensity, and scapulothoracic 3D kinematics, were post-processed with TheMotionMonitor software (Innsport, Chicago IL) and results average for each condition.

Results: No statistical significant differences were found in both tasks, when comparing the UT and LT activation intensity and onset results, with and without the ST. A statistical significant $(p<0.01)$ increase in scapular posterior tilt was observed in both tasks when the ST was applied.

Discussion: The results of this study revealed that ST has a direct mechanical effect on scapula motion, in particularly, an increase in scapula posterior tilt. The assumption that this taping technique induces an inhibition of the UT and a facilitation of LT could not be corroborated based on our results. Patients with shoulder pain frequently present a decreased scapular posterior tilt, which is believed to be a contributor factor to the shoulder dysfunction. Rehabilitation programmes aiming to restore scapular motion may benefit from the application of ST.

\section{References}

[1] Kibler. Br. J. Sports Med. 2013;47:877-85.

[2] McConnell, Coursenotes - The McConnell Institute.

\footnotetext{
* Corresponding author.

E-mail address: ftanaclemente@gmail.com (A. Clemente).
} 